\title{
Atypical HUS: time to take stock of current guidelines and outcome measures?
}

\author{
Lesley Rees
}

Received: 27 December 2012 / Revised: 19 January 2013 / Accepted: 21 January 2013 /Published online: 7 February 2013

(C) IPNA 2013

\begin{abstract}
European guidelines for the assessment and management of atypical HUS were written in 2009. Since then our understanding of this group of diseases has advanced. Evidence is emerging that eculizumab, a monoclonal antibody inhibiting $\mathrm{C} 5$ activation, is effective, and potentially superior to current treatment with plasmapheresis. The evidence base for the benefits of plasmapheresis consists of case series and small reports. Before we embark on a change of management policy it is vital that we set up a system for genetic diagnosis, standardised protocols and a means to collect predetermined outcome measures, so that we do not make the same mistakes with assessment of the effectiveness of eculizumab as we did for plasmapheresis.
\end{abstract}

Keywords Hemolytic uremic syndrome .

Thrombocytopenia · Acute kidney injury

\section{Introduction}

The term haemolytic uraemic syndrome (HUS) is a descriptive expression for patients presenting with a combination of microangiopathic haemolytic anaemia, thrombocytopaenia and acute kidney injury. It is not, therefore, just as for 'nephrotic syndrome' or 'glomerulonephritis', a diagnosis in itself, but rather a collection of diseases with differing causes, presentations and outcomes. In addition, it is rare, making clinical trials and studies of outcome fraught with the difficulties of small patient numbers and biases incurred by disease variability. So how do we decide the best treatment strategies

L. Rees $(\bowtie)$

Great Ormond Street Hospital, London, UK

e-mail: I.rees@ucl.ac.uk for patients with atypical HUS (aHUS) and how do we assess outcomes with such diverse aetiologies?

\section{Background}

Traditionally, HUS has been divided into diarrhoea-positive (D+) and diarrhoea-negative (D-), the former resulting from verocytotoxin-producing Escherichia coli and Shigella dysenteriae type 1, the latter encompassing all other causes. In view of the unreliability of diarrhoea as a distinguishing feature, current terminology used to describe HUS divides it into 'typical' for that due to infective causes and 'atypical' (aHUS) for other causes. Typical HUS is more common, with verocytotoxin-producing organisms being the commonest aetiology, although the incidence of Pneumococcal HUS is increasing so that in some countries it is overtaking verocytotoxin-associated HUS as the commonest cause. aHUS has an incidence of only two per million population per year, or seven per million child population, and accounts for approximately $5 \%$ of the total cases of HUS [1]. There are several different aetiologies: it may be secondary to autoimmune diseases or occur post transplantation or in association with drugs. However, the most difficult to manage is aHUS that is associated with genetic disorders of regulation of the alternative pathway of complement. This is because such mutations can cause devastating kidney injury or even death, or can lead to a chronically relapsing course.

\section{The diversity of aHUS due to genetic mutations}

To date, genetic mutations can be identified in approximately $50 \%$ of patients with primary aHUS, and more are 
continuously being sought and identified. Some mutations are more common than others, and prognosis also varies according to the mutation. For example, abnormalities of complement Factor $\mathrm{H}$ (the most important regulator of the alternative pathway) are the most frequent findings, explaining about $30 \%$ of cases, and have the worst prognosis, with a $60-70 \%$ incidence of chronic kidney disease (CKD) within 5 years or death within 1 year, whereas mutations in membrane cofactor protein (MCP; CD46), which protects cells from damage from complement, have the best outcome, with more than $80 \%$ of patients remaining dialysis independent, although recurrent episodes are common. Some children can have more than one mutation. Furthermore, most abnormalities are heterozygous, and there can be incomplete penetrance such that first degree relatives carrying the same mutation are often asymptomatic. Therefore, presumably, for aHUS to develop there must be a combination of genetic and environmental factors [2]. In other words, the detection of genetic abnormalities provides a risk indicator but is not a good marker alone. We need to focus our research on identifying biomarkers for these diseases and developing metrics for measuring environment triggers.

\section{Current guidelines for management of aHUS}

In 2009, The European Study Group for Atypical HUS published guidelines for the initial assessment and early management of children with aHUS [3]. It is stated from the outset that this is a consensus document rather than one based on sound evidence. The protocol recommended is plasmapheresis within $24 \mathrm{~h}$ of diagnosis with whole plasma fraction replacement fluid [fresh frozen plasma or virus-inactivated pooled plasma (e.g. Octaplas $\left.{ }^{\circledR}\right)$ ]. Plasmapheresis is then to be repeated daily for 5 days, followed by five sessions per week for 2 weeks, then three sessions per week for 2 weeks. There is no further advice for management beyond this point, although most centres would attempt withdrawal of plasmapheresis if remission has occurred, with variable protocols, but monitoring for relapse and reinstitution if withdrawal fails. The conclusion to the guidelines is that they should be rewritten after 3 years. So, what have we learnt, what new developments have there been in the meantime and are we in a better position now to assess treatment success than we were in 2009 ?

\section{Is plasmapheresis effective?}

Do we know whether plasmapheresis is an effective treatment, and who would we expect and who would we not expect to benefit from it? The underlying rationale of this treatment is to remove circulating mutated factors or factor $\mathrm{H}$ antibodies and to replace deficient ones, such as ADAMTS or complement regulatory factors. Plasmapheresis would not be expected to benefit patients with MCP deficiency as this is a membranebound factor rather than a circulating one. Outcomes have recently been reviewed $[4,5]$. However, most reports stem from the days before genetic identification and stratification. Overall, although around $60 \%$ of patients with aHUS respond initially [6], reports suggest that there is a $25 \%$ mortality rate and that $50 \%$ are left with CKD [3]. Furthermore, plasmapheresis, although a safe procedure with a mortality of less than 1 in 10,000 [5], is not without its complications: there are risks associated with the insertion of vascular access, access infection, fluid disturbances during the process itself, hypersensitivity reactions, as well as a very small risk of viral transmission from plasma.

\section{Eculizumab}

The most exciting development in the management of aHUS is eculizumab (Soliris ${ }^{\circledR}$, Alexion Pharmaceuticals, Cheshire, $\mathrm{CT}$ ), a monoclonal antibody licenced for use in aHUS that targets $\mathrm{C} 5$ and, therefore, the ultimate point in the alternative pathway cascade. There have been small series and case reports of patients who have been successfully treated with eculizmab since the 2009 guidelines were published, along with multicentre prospective phase II trials in adults and adolescents and an ongoing paediatric trial. Indeed, there are reported cases of children responding to eculizumab when plasmapheresis has failed [6]. In this edition of Pediatric Nephrology, two children are described who had severe neurological involvement unresponsive to plasmapheresis, but who both responded to eculizumab [7]. The drug has been used for over 10 years in patients with paroxysmal nocturnal haemoglobinuria and has been demonstrated to have very few side effects, although the doses used for this disease are about $30 \%$ lower than those currently recommended for aHUS [4]. All patients need to be vaccinated against Neisseria meningitidis before starting treatment, and many physicians use antibiotic prophylaxis as well. What we do not know is when or whether eculizumab can be withdrawn after remission as there are reports of the development of severe relapse in this situation [8]. The European Medicines Agency has approved life-long therapy.

\section{Should the guidelines for management of aHUS be updated?}

We are now 3 years further on and it is time to update the European guidelines. At what point do we decide that case series or individual reports demonstrating benefit are 
sufficient to justify following a new treatment plan? Many clinicians now believe that eculizumab should be the new standard of care for children with aHUS [2, 3, 6]. Should it be first line treatment or should it only be used after failure of plasmapheresis? The expense of the drug has to be taken into consideration, but part of the economics has to include the social and financial aspects of saving a patient from dialysis and transplantation. Are we now in a better position to assess the benefits of eculizumab than we were for assessing those of plasmapheresis?

\section{Conclusion}

Before we embark on widespread changes of management policy we need to establish that certain criteria are in place to monitor the effects of our changes. First of all we need to have a system for rapid genetic screening and diagnosis to enable stratification of outcomes according to mutation analysis. Secondly, as genetic abnormalities alone are not the whole answer to disease risk, severity and outcome, we need to find other biomarkers (such as functional complement pathway assays) and determinants of environmental triggers. Thirdly, we must decide upon our definitions for success. Clear outcome measures are death and need for ongoing dialysis, but there may be a range of morbidities, such as proteinuria, hypertension, varying grades of CKD and relapses, over an unpredictable time course. Finally, we must decide what our treatment protocols will be, whether all children will be treated with the same protocol, and, if treatment is successful, how long it will be continued for. Should a strategy for treatment withdrawal be part of any protocol? Most important of all, the development of registries for aHUS will be critical, but data to determine outcome will only be useful if genetic testing is available and standardized treatment regimens and outcome definitions are used to enable useful analysis.

Conflict of interest The author has received educational grants from Alexion.

\section{References}

1. Taylor CM, Machin S, Wigmore SJ, Goodship THJ, on behalf of a working party from the Renal Association, the British Committee for Standards in Haematology and the British Transplant Society (2009) Clinical practice guidelines for the management of atypical haemolytic uraemic syndrome in the United Kingdom. Br J Haematol 148:37-47

2. Bu F, Borsa N, Gianluigi A, Smith RJH (2013) Familial atypical hemolytic uremic syndrome: a review of its genetic and clinical aspects. Clin Devel Immunol. doi:10.1155/2012/370426

3. Ariceta G, Besbas N, Johnson S, Karpman D, Landau D, Licht C, Loirat C, Pecoraro C, Taylor MC, Van de Kar N, VandeWalle J, Zimmerhackl LB, The European Paediatric Study Group for HUS (2009) Guideline for the investigation and initial therapy of diarrhea-negative hemolytic uremic syndrome. Pediatr Nephrol 24:687-696

4. Loirat C, Frémeaux-Bacchi Orphanet V (2011) Atypical hemolytic uremic syndrome. J Rare Dis 6:60

5. Clark WF (2012) Thrombotic microangiopathy: current knowledge and outcomes with plasma exchange. Semin Dial 25:214-219

6. Zuber J, Fakhouri F, Roumenina LT, Loirat C, Fremeaux-Bacchi V, on behalf of the French study group fo aHUS/C3G (2013) Use of eculizumab for atypical HUS and C3 glomerulopathies. Nat Rev Nephrol 8:643-657

7. Gulleroglu K, Fidan K, Hançer VS3, Umut Bayrakci U, Baskin E, Soylemezoglu O (2013) Neurologic involvement in atypical hemolytic uremic syndrome and successful treatment with eculizumab. Pediatr Nephrol. doi:10.1007/s00467-013-2416-9

8. Barbour T, Johnson S, Cohney S, Hughes P (2102) Thrombotic microangiopathy and accosiated renal disorders. Nephrol Dial Transplant 27:2673-2685 